Primary mediastinal large B-cell lymphoma has been recognised as a distinct entity with unique clinical pathologic, and genetic features. According to WHO 2008 classification it is marked as a variant of diffuse large B-cell lymphoma but shares characteristics with classic Hodgkin lymphoma. Genetic analysis has shown that amplification of the 9p24.1 region is the disease's specific structural alteration. Aggressive behaviour and a tendency to invade surrounding tissues of the thoracic cavity, often causing superior vena cava syndrome, or pleural or pericardial effusions, are the clinical hallmarks of this disease. For a long period of time it has been considered as a disease with poor prognosis, which responds poorly to the conventional treatment created for diffuse large B-cell lymphoma. An elective treatment has not yet been established, but recently the situation has became much more favourable. After the introduction of rituximab the cure rates have risen to over $80 \%$, and the most recent results have demonstrated a new insight with dose-adjusted intensified continuous treatments, in which the cure rates have exceeded $90 \%$. Current trends have led to the introduction of dose-adjusted intensified protocols becoming a standard of care, whereas the use of radiotherapy remains controversial because of the questionable predictive value of post-treatment PET/CT validity. The relapse rate is very low after two years of sustained complete remission. If the disease relapses or is resistant the outcome is very poor regardless of the applied treatment modality.

Key words: primary mediastinal large B-cell lymphoma, immunochemotherapy, radiotherapy, treatment options.

Contemp Oncol (Pozn) 2015; 19 (6): 428-435 DOI: $10.5114 /$ wo.2015.56388

\section{Current trends in the treatment of primary mediastinal large B-cell lymphoma - an overview}

\author{
Ivan Petković
}

Oncology Clinic, Clinical Centre Niš, Serbia

\section{Introduction}

The first papers referred to large cell lymphoma accompanying prominent fibrosis, which affects predominantly the mediastinum and has a propensity to invade the superior vena cava, especially in younger women, were published in the early 1980s [1-3]. The first recognition of the entity as a separate one was appointed by revised European-American classification of lymphoid neoplasms in 1994 [4, 5]. The latest revision of World Health Organization (WHO) 2008 classification of lymphoid neoplasms included it in the category of other lymphomas of large B-cells, as a variant of the diffuse large B-cell lymphoma (DLBCL) [6]. It is marked as a distinct entity with unique clinical, pathologic, and genetic features under the name primary mediastinal large B-cell lymphoma (PMLBCL).

Microarray studies revealed a unique molecular signature of $P M L B C L$, distinguishing it from DLBCL, with a striking overlap with the nodular sclerosis subtype of classical Hodgkin lymphoma ( $\mathrm{NSCHL})[7,8]$. Indeed, both neoplasms share certain clinical, histologic, and molecular features. Nodular sclerosis subtype of classical Hodgkin lymphoma and PMLBCL are composed of large somewhat polymorphous cells immersed in pleomorphic inflammatory infiltrate commonly associated with compartmentalising alveolar fibrosis $[6,9]$. Amplification of the 9p24.1 region is a disease-specific structural alteration that increases both the gene dosage of programmed cell death ligand-1 (PD-L1) and it includes the janus kinase 2 locus (JAK2). Of note, JAK2 amplification increased protein expression and activity, it specifically induced PD-1 ligand transcription, and it enhanced sensitivity to JAK2 inhibition [9]. These aberrations have been suggested to affect tumour-microenvironment interactions resulting in immune privilege [10]. Recent work has identified PMLBCL disease biology as dependent on molecular pathways involving REL, JAK-STAT, PD-L1/PD-L2, and nuclear factor $\kappa \beta(N F \kappa \beta)$ [11]. In the future these might be rational therapeutic targets. Primary mediastinal large B-cell lymphoma cells express pan B-cell antigens (CD19, CD20, CD22, and CD79 $\alpha$ ), while CD30 is present in more than $80 \%$ of cases, although usually weak and heterogeneous compared to $\mathrm{CHL}$. Large B-cells are frequently positive for IRF4/MUM1 (75\%) and CD23 (70\%), and have variable expression of bcl2 (55-80\%) and bcl6 (45-100\%), while CD10 is less common, so the immunophenotype is usually of non-germinal centre origin (non-GCB) [6].

Primary mediastinal large B-cell lymphoma comprises approximately $2-4 \%$ of all non-Hodgkin lymphomas ( $\mathrm{NHL}$ ) [6] and $6-10 \%$ of all DLBCL [12], typically occurring in young adults in their third to fourth decade of life, with a female predominance [13].

Clinically, PMLBCL presents mostly as a "bulky" tumour of the anterior mediastinum with rapid progression and locally compressive effects including dyspnoea, cough, dysphagia, hoarseness, and superior vena cava syndrome in up to half of patients. Bone marrow infiltration at presentation is 
rare. Extranodal sites, however, may be involved, particularly at the time of disease recurrence.

Selection of the optimal induction treatment for PM$L B C L$ is a matter of great controversy. Numerous studies have pointed out the importance of achieving remission with front-line treatment since it gives an opportunity to overcome the disease. Thus, wisdom is required in making the decision whether treatment approach to choose to initiate treatment. R-CHOP (rituximab, cyclophosphamide, doxorubicin, vincristine, and prednisone) could be considered as de facto standard, but it is not universally accepted [14]. The so-called third-generation protocols: MACOP-B/ VACOP-B (methotrexate, doxorubicin, cyclophosphamide, vincristine, prednisone-bleomycin/etoposide instead of methotrexate and the same drugs as indicated above) mostly showed an advantage regarding complete remission (CR) rates and progression-free survival (PFS), but overall survival (OS) was not significantly better even after involving rituximab. Some of the most recent publications have pointed towards the protocols with dose-adjusted high-dose induction having a high impact on event-free survival (EFS) and OS and have marked it as an advanced treatment for PMLBCL. The role of consolidation with radiotherapy (RT) remains controversial. However, by introducing PET/CT guided response evaluation using the Deauville scoring system (Table 1) it is becoming clearer which group of patients might benefit from the addition of RT after initial treatment. Relapsed/refractory disease is associated with extremely poor outcome in most cases, regardless of the applied treatment modality.

\section{General considerations of front-line management to primary mediastinal large B-cell lymphoma}

Primary mediastinal large B-cell lymphoma was initially considered as a disease with a relatively high proportion of primary refractoriness, early relapse, or unsuccessful treatment after standard anthracycline-containing induction (CHOP-like regimens) [15-17]. Induction treatment of PMLBCL is not optimally defined, but most reports suggest that immunochemotherapy alone is inadequate and the use of routine mediastinal consolidation RT is required. On the other hand, some novel reports suggest that dose intensification of front-line treatment gives a greater chance to improve long-term results, and they question the routine use of consolidation with RT. Nevertheless, if induction therapy achieves $C R$, late relapses are only rarely observed.

The aim of this paper is to review the most recent literature up-dates on the treatment of PMLBCL.

\section{Review of chemotherapy selection in front-line approach}

The majority of analysed PMLBCL case series are based on retrospective observations. A retrospective observation of 138 patients, comparing CHOP to MACOP-B/VACOP-B, published by Todeschini et al., found that the advantages were statistically significant in the MACOP-B/VACOP-B-treated group at low/low-intermediate risk $(p=0.001)$. In the small group of high-intermediate/high-risk patients, an advan-
Table 1. Deauville criteria* and scoring system

\begin{tabular}{|c|c|}
\hline \multicolumn{2}{|c|}{ Deauville score } \\
\hline 1 & no FDG uptake \\
\hline 2 & FDG uptake $\leq$ mediastinum \\
\hline 3 & FDG uptake $>$ mediastinal but $\leq$ liver \\
\hline 4 & FDG uptake > liver at any site \\
\hline 5 & FDG uptake > liver and new sites of disease \\
\hline$x$ & $\begin{array}{l}\text { new areas of FDG uptake unlikely to be related to } \\
\text { lymphoma }\end{array}$ \\
\hline \multicolumn{2}{|c|}{$\begin{array}{l}\text { *Visual interpretation of the PET-CT scan uses a 5-point scale (i.e. Deauville cri } \\
\text { teria). } \\
\text { A score of 1-3 was regarded as negative and } 4 \text { or } 5 \text { as positive. } \\
\text { This scale has been used effectively in Hodgkin lymphoma (HL), but in DLBCL } \\
\text { the results need to be clarified. }\end{array}$} \\
\hline
\end{tabular}

tage from MACOP-B/VACOP-B as compared to CHOP was observed in terms of CR rate and EFS, but it was not statistically significant $(p=0.068)$. The achievement of CR was the most significant prognostic factor both for OS and EFS $(p<0.0001)$. A high percentage of patients who reached CR remained event-free, suggesting that a therapeutic approach able to increase the CR rate could influence the long-term OS and EFS. In multivariate analysis, CR achievement and type of chemotherapy (MACOP-B, VACOP-B) were independent prognostic factors for EFS, while the International Prognostic Index (IPI) (Table 2) did not prove to be significant [18]. The above-mentioned results are in agreement with many previously published retrospective studies [19-24]. A retrospective observation of the British Columbia database single-centre experience $(n=153)$ indicated that patients with PMLBCL (base collection from 1980 to 2003) and treatment

Table 2. International Prognostic Index (IPI)

\begin{tabular}{lcc} 
Age & $\leq 60$ years & 0 \\
Ann Arbor clinical stadium & $>60$ years & 1 \\
ECOG performance status & I and II & 0 \\
& III and IV & 1 \\
Serum LDH activity & 0 or 1 & 0 \\
& $2-4$ & 1 \\
Extranodal involvement & normal & 0 \\
& $\leq 1$ extranodal site & 0 \\
\hline
\end{tabular}

IPI 0-1 - low; IPI 2-low/intermediate; IPI 3-high/intermediate; IPI 4-5 - high risk

Table 3. Age-adjusted International Prognostic Index (aalPI)

\begin{tabular}{|c|c|c|}
\hline \multirow[t]{2}{*}{ Ann Arbor clinical stadium } & I and I| & 0 \\
\hline & III and IV & 1 \\
\hline \multirow[t]{2}{*}{ Serum LDH activity } & normal & 0 \\
\hline & elevated & 1 \\
\hline \multirow[t]{2}{*}{ Extranodal involvement } & $\leq 1$ extranodal site & 0 \\
\hline & $>1$ extranodal site & 1 \\
\hline
\end{tabular}

aaIPI 0 - low; aalPI 1 - low/intermediate; aalPI 2 - high/intermediate; aalPl 3 - high risk 
modalities (1980-1992 MACOP-B/VACOP-B; 1992-2001 CHOP-type; 2001-2003 CHOP-R) demonstrated a favourable outcome of PMLBCL patients in comparison to DLBCL. They noted also that "age-adjusted" IPI (aalPI) (Table 3) was not predictive of survival, suggesting other prognostic modalities to be suitable for risk stratification. Dose-intensified chemotherapy MACOP-B/VACOP-B demonstrated a trend towards superior outcome over CHOP-type chemotherapy [14]. The latter publication of Zinzani et al., as well retrospective analysis of 45 patients previously untreated with PMLBCL, who received MACOP-B or VACOP-B + rituximab and mediastinal RT, showed that 26 (62\%) patients achieved CR, and 15 (36\%) obtained a partial response after MACOP-B/VACOP-B + rituximab. The projected OS for this study (with RT addition) was $80 \%$ at five years. In comparison with historical data on MACOP-B/VACOP-B and no rituximab, there were no statistical differences in terms of CR and relapse-free survival (RFS) rates [25]. Retrospective studies have long suggested that patients with PMLBCL have improved outcomes with the receipt of regimens of increased dose intensity [24]. One of the explored dose-intensity protocols for the treatment of high-risk DLBCL and PMLBCL is DA-EPOCH (dose adjusted-etoposide, doxorubicin, vincristine as a 96-hour continuous infusion + cyclophosphamide and prednisone) chemotherapy regimen. It is a doxorubicin-containing schedule, which allows the delivery of high maximal cumulative doses of the drug without clinically significant cardiac toxic effects [26, 27]. Dose-adjustment is mostly based on the decrease of absolute neutrophil or platelet count. It leads to inhibition of topoisomerase II and down-regulation of bcl6 expression, suggesting that regimens directed against topoisomerase II might have increased efficacy in treating PMLBCL. In this regard, DAEPOCH-(R) was designed to inhibit topoisomerase $\|$ by including two topoisomerase II inhibitors, etoposide and doxorubicin, and maximising topoisomerase II inhibition by way of extended drug exposure [28]. Outcomes associated with the use of DA-EPOCH-R may be related to dose intensity as well as the continuous infusion schedule [29].

\section{The role of rituximab addition to front-line chemotherapy treatment}

Significant data about the effects of rituximab in DLBCL patients was obtained from the results of the MabThera International Trail (MInT). The study observed 824 patients with good prognosis DLBCL (aged $<60$ years, stage I "bulky", or stage II-IV, with aalPI score 0-1). The aim was to compare rituximab addition to chemotherapy vs. chemotherapy alone. Patients assigned chemotherapy and rituximab had increased three-year EFS compared with those assigned chemotherapy alone (79\% vs. 59\%, $p<0.0001)$, and had increased three-year OS (93\% vs. $84 \%$, $p=0.0001$ [30]. Later published results of 87 patients drawn from a total of 824 enrolled in MInT were those with PMLBCL. These results showed that rituximab increased the rates of CR (unconfirmed) in both PMLBCL (from 54\% to $80 \%, p=0.015$ ) and DLBCL (from $72 \%$ to $87 \%, p<0.001$ ). In PMLBCL, rituximab virtually eliminated progressive disease (PD) $(2.5 \%$ vs. $24 \%, p<0.001)$, whereas without rit- uximab, PD was more frequent in PMLBCL than in DLBCL ( $24 \%$ vs. $10 \%, p=0.010$ ). With a median observation time of 34 months, three-year EFS was improved by rituximab for PMLBCL (78\% vs. 52\%, $p=0.012)$ and for DLBCL (81\% vs. $61 \%, p<0.001)$. Overall survival benefit was similar for DLBCL (93\% vs. $85 \%, p<0.001)$ and PMLBCL ( $89 \%$ vs. $78 \%$, $p=0.158$ ) [31]. The main conclusion was that in young patients with PMLBCL (aalPI 0-1) R-CHOP is an effective treatment with favourable outcome. However, the presented results do not refer to the subgroup of patients with aalPI $>1$ (high-intermediate or high risk aalPI, rate from 22\% to $59 \%$ in various studies), which MInT did not examine [30]. Vassilakopoulos et al. published one of the largest prospective serious of PMLBCL patients, regardless of aalPI score. The study compared R-CHOP $\pm R T$ in 76 patients with historical control of $\mathrm{CHOP} \pm \mathrm{RT}$ alone in 45 patients. The results showed that irrespective of aalPI score, the five-year freedom from progression (FFP) rate after $\mathrm{R}-\mathrm{CHOP} \pm \mathrm{RT}$ was $81 \%$ vs. $48 \%$ for $\mathrm{CHOP} \pm \mathrm{RT}(p<0.0001)$. The five-year EFS rates were $80 \%$ and $47 \%(p<0.0001)$ and the five-year OS and lymphoma-specific survival rates were $89 \%$ and $69 \%$ ( $p=0.003)$ and $91 \%$ and $69 \%(p=0.001)$, respectively, with only 7 of 76 lymphoma-related deaths [32]. A Korean group concluded in a prospective cohort of 21 patients that the two-year OS of the R-CHOP arm was superior to a historical cohort of 14 patients treated with CHOP (82.7\% vs. $57.1 \%)$, but statistical significance of this survival benefit was not reached ( $p=0.081)$. Nevertheless, their comparison suggested that R-CHOP might increase the response and reduce relapses, resulting in prolongation of PFS [33]. The Magyar group published their results of a cohort of 20 newly diagnosed PMLBCL patients treated with R-CHOP and compared them with historical control patients $(n=9)$ treated with $\mathrm{CHOP}$ alone and 15 patients treated with ProMACE-CytaBOM (prednisone, doxorubicin, cyclophosphamide, etoposide, cytarabine, bleomycin, vincristine, methotrexate, and leucovorin). Comparing the results of $\mathrm{R}-\mathrm{CHOP}$ with highly aggressive ProMACE-CytaBOM they found similar results with statistical differences in the order of five-year OS: $79.4 \%$ vs. $80 \%$; EFS: $70 \%$ vs. $6 \%$; RFS: $93 \%$ vs. $82 \%(p>0.05)$. Adding rituximab to CHOP therapy gave results as good as with high aggressive protocols. The main conclusion was that highly toxic protocols could be safely replaced with less toxic R-CHOP, which offers almost the same treatment results [34]. The recent reports of Chinese experts from 2013 analysed cohort of 79 PML$B C L$ patients and demonstrated the significant superiority in survival benefit of R-CHOP $(n=39)$ regimen over $\mathrm{CHOP}$ ( $n=40$ ) alone. Patients assigned to the rituximab arm had five-year OS and PFS rates of $>83 \%$ and $>76 \%$, compared with $>48 \%$ and $>44 \%$ for CHOP, respectively. Early stage disease patients had five-year OS and PFS rates of 93.8\% and $84.6 \%$ with R-CHOP, and $52.0 \%(p=0.002)$ and $46.6 \%$ ( $p=0.003$ ) with $\mathrm{CHOP}$, respectively [35]. The most recently published reports, from a Chinese group of experts, also demonstrated their retrospective results with R-CHOEP (R-CHOP + etoposide) as a front-line therapy vs. CHOEP regimen. The cohort included 29 patients with PMLBCL, median age 32 years, with median follow up of 29 months. Two patients failed to be followed. Among the 27 
evaluable patients, 17 achieved CR, five achieved $P R$, one relapsed, and four died of PD. The five-year OS was $85.2 \%$, in which R-CHOEP regimen group patients had OS 94.4\% and CHOEP group patients had OS 75\%, eight patients underwent autologous stem cell transplantation (auto-SCT) and one patient underwent allogeneic stem cell transplantation (allo-SCT), who remained in CR state. R-CHOEP chemotherapy regimen can achieve satisfactory results but needs to be explored by further clinical trials [36]. We did not find any trial that had compared directly R-CHOP with R-CHOEP regimen. The National Cancer Institute group presented their preliminary results in 2008 at Lugano Conference, based on 40 patients who had been enrolled since that moment, with no additional RT in 39, that FFS and OS rates were $93 \%$ and $100 \%$, respectively, significantly higher in the DA-EPOCH + rituximab arm than in the DA-EP$\mathrm{OCH}$ historical control arm [37]. A few years later the same group published updated results of the same study, now conducted on 51 patients as a phase II single-group prospective trial of DA-EPOCH-R and filgrastim as induction regimen, without the use of RT. The control group enrolled 16 patients retrospectively observed from another centre, treated with the same protocol, also without RT. In the follow-up period of five years (maximum $>13$ years) the EFS rate was $93 \%$ and the OS rate was $97 \%$. Despite phase II study and retrospective observation the results formalised that rituximab significantly improves outcome when added to a DA-EPOCH regimen, suggesting that it represents an advance in the treatment of PMLBCL [38]. The Spanish PETHEMA group presented their prospective multi-institutional phase II study of the long-term follow-up of DA$\mathrm{EPOCH}+\mathrm{R}$ in untreated patients with poor prognosis large B-cell lymphoma. Most of the patients involved had $\operatorname{DLBCL}(n=68)$ and PMLBCL $(n=6)$. Interestingly, patients with bcl6 rearrangement achieved a 10 -year OS of $100 \%$, while patients with bcl2 rearrangement exhibited a poorer outcome compared to activated B-cell tumours and germinal centre B-cell without bcl 2 rearranged tumours. Results achieved with DA-EPOCH-R showed a good long-term outcome and a tolerable toxicity profile in high-risk large $B$ cell lymphoma patients [39]. The most recent publication of a retrospective cohort of 95 patients, which compared R-VACOP-B VS. R-CHOP with historical controls with no rituximab and a predictive value of interim FDG-PET/CT evaluation, demonstrated that the default superiority of the third-generation regimen disappeared after the inclusion of rituximab [40]. In one of the most recent results from single-centre observational retrospective study on a cohort of 74 patients with PMLBCL treated with R-MACOP-B presented by Zinzani et al., it was demonstrated that 61 (82.4\%) patients achieved CR after the R-MACOP-B regimen; $68.9 \%$ presented a positive final PET and were treated with local RT; and $31.1 \%$ had a negative PET. Five patients relapsed within 12 months. At 10 years, OS was $82 \%$, PFS was $87.6 \%$, and DFS was $90.5 \%$ (median follow-up four years). No statistically significant differences were observed in DFS between the patients treated also with RT (PET positive) and patients only observed (PET negative): $90.7 \%$ vs. $90 \%$ ( $p=0.85)$, respectively. The main conclusion of the study was that adding rituximab does not change the final results in terms of complete response and DFS utilising a third-generation regimen [41]. Recent remarks by Prof. Zinzani and his group launched several controversial issues about the superiority of third-generation regimens and the impact of rituximab.

Considering all the evidence presented, it can be concluded that adding rituximab to the selected group of chemotherapeutic regimens (CHOP/CHOP-like or DA-EPOCH) significantly improves treatment outcome of patients with PMLBCL, while the results with the third-generation protocols (MACOP-B/VACOP-B) are controversial and need to be clarified in future prospective trials. A summarised review of prospective trials is given in Table 4.

\section{Consolidation therapy}

Whether to incorporate the consolidation therapy after the completion of induction treatment with immunochemotherapy depends on many variables: was the applied immunochemotherapy effective enough to fully suppress the disease itself or not, what is the real predictive value of FDG-PET/CT scan positivity in interim analysis, and is the residual FDG-PET/CT scan positivity predictive enough for the disease rest after completion of the induction? In that order RT can be considered as consolidation to primary treatment in order to reinforce achieved remission or as an effort to convert and improve the insufficient effects of induction treatment to remission.

\section{The role of consolidation with radiotherapy}

Contradictory data regarding the use of consolidative RT after post treatment PET/CT scan in PMLBCL have been obtained. However, it seems that things are becoming clearer with some novel reports of PET/CT utilisation as a predictive tool for a tailored approach to RT consolidation. This topic will be discussed. On the other hand, the

Table 4. Recent prospective trials investigating the role of the front-line therapies in PMLBCL

\begin{tabular}{|c|c|c|c|c|c|}
\hline Reference & $\begin{array}{c}\text { Number of } \\
\text { patients enrolled }\end{array}$ & $\begin{array}{c}\text { Type of front-line } \\
\text { therapy }\end{array}$ & $\begin{array}{l}\text { Event free-survival } \\
\text { rate (EFS) (\%) }\end{array}$ & $\begin{array}{l}\text { Overall survival rate } \\
\text { (OS) (months) (\%) }\end{array}$ & $\begin{array}{l}\text { Median follow-up } \\
\text { period }\end{array}$ \\
\hline Ahn et al. [33] & 21 & R-CHOP & 79 & 82.7 & 2-years \\
\hline Schneider et al. [34] & 20 & R-CHOP & 70 & 79.4 & 5-year \\
\hline Rieger et al. [31] & 87 & $\mathrm{R}-\mathrm{CHOP}$ & 78 & 89 & App 3-year \\
\hline Vassilakopoulos et al. [32] & 76 & R-CHOP & 80 & 89 & 5-year \\
\hline Xu et al. [35] & 39 & $\mathrm{R}-\mathrm{CHOP}$ & 76.3 & 83.7 & 5-year \\
\hline Dunleavy et al. [38] & 51 & DA-EPOCH-R & 93 & 97 & 5-year \\
\hline
\end{tabular}


cited prospective phase II study of Dunleavy et al., questioned the objective significance of post-treatment FDG/ PET scan follow up, suggesting that the technique has a poor positive predictive value (PPV) in PMLBCL. Independent verification of their study concluded that 2 of 51 patients (4\%) actually had a need for consolidative RT. The general conclusion was that DA-EPOCH-R has a high cure rate and obviates the need for consolidative RT in 49 (96\%) patients with PMLBCL [38]. Another recent, admittedly retrospective observation of $95 \mathrm{pa}-$ tients, which investigated the role of interim FDG-PET/CT scan predictive values during induction with R-VACOP-B, showed that PPV of FDG-PET/CT were small, as opposed to its negative predictive values (NPV). The conclusion was that using the FDG-PET/CT scan as a guide for continuation of induction treatment remains unclear due to its relatively low PPV [40]. Nevertheless, those novel reports are a matter of a great debate among experts, and it raises the question of future RT emplacement as a treatment modality for PMLBCL. Following reports from a variety of studies, contradictory data about the objective relevance of RT were obtained. The conclusion of a comprehensive retrospective British-Columbia single-centre experience $(n=153)$ was that the routine use of RT does not improve survival [14]. Another, also retrospective, observation of 45 patients showed that RT was able to convert the effect of immunochemotherapy of CR additionally for approximately 20\% (MACOP-B/VACOP-B + R achieved $62 \%$ of CR, and with the addition of RT it was $80 \%$ ) [25]. In this regard, the most recent publication of Zinzani et al., demonstrated that the use of additional RT after induction with an R-MACOP-B regimen enhance the results achieved only by induction therapy [41]. Based on Rebecca Kirk statements it seems that in most of the patients, immunochemotherapy is not curative, and consolidation RT is offered [42]. Early stage PMLBCL patients treated with R-CHOP and RT showed 5-year OS, PFS and local control (LC) rates of $96.4 \%, 85.9 \%$ and $93.1 \%$, respectively. R-CHOP + consolidation RT was associated with excellent survival and LC rates in this specific subgroup of patients [35]. In prospective study cohort of $(n=37)$ patients, where all received immunochemotherapy and were post-treatment FDG-PET/CT scan evaluated, and all had residual activity. The three-year OS and PFS of the whole cohort were $89.8 \%$ and $88.7 \%$, respectively. Overall survival was significantly different between scores 1-3 and scores 4-5 (100\% vs. $77 \%$ at three years, $p<0.05)$. Approximately $50 \%$ of PMLBCL patients showed residual disease at FDG-PET scan after immunochemotherapy. Radiotherapy was found to be able to convert to CR in approximately $85 \%$ of these patients, but only those with a Deauville score of 5 (10\%) appeared to be at high risk of progression and death, and they might be candidates for intensified programs [43]. The most recently published prospective study by Martelli et al., showed that PET/CT has a prognostic impact and can predict survival after immunochemotherapy. They evaluated 125 patients, and 115 had central review of PET/ CT scans by using five-point scale according to Deauville. Consolidation RT was permitted and given to 102 patients. The results showed that using the liver uptake as a cut-off for PET positivity (boundary of score 3 to 4) discriminated most effectively between high or low risk of failure, with a five-year PFS of $99 \%$ vs. $68 \%(p<0.001)$ and five-year OS of $100 \%$ vs. $83 \%(p<0.001)$. This study provides a basis for using PET/CT to define the role of RT in PMLBCL [44]. Longterm consequences of thoracic RT after prior exposure to chemotherapy are well established, especially in the context of its use in mediastinal presentation HL. Similar data regarding the combined use of immunochemotherapy + RT in PMLBCL patients does not exist. However, it can be assumed that the effects would probably be the same.

\section{The role and place of stem cell transplantation}

Given the excellent results obtained with present new induction regimens $\pm R T$, the role of front-line use of auto-SCT is considered redundant. GEL-TAMO registry presented 71 patients with PMLBCL receiving induction chemotherapy followed by auto-SCT in the front-line setting. Most of the patients had high-risk clinical features. At transplant, $49 \%$ of patients were in CR, 32\% in PR, and $18 \%$ failed induction therapy; $53 \%$ received RT. After the transplant $75 \%$ of patients reached CR. With a median follow-up of 52.5 months, the OS, PFS, and DFS at four years from diagnosis were, respectively, $84 \%, 81 \%$, and $81 \%$ for the first CR patients and $49 \%, 42 \%$, and $82 \%$ for the induction failure (PR and refractory) patients. Disease progression was the main cause of death (79\%). By multivariate survival analysis of the tumour score, refractory disease at transplant and RT were independent variables associated with OS and PFS. The GEL-TAMO experience showed that with a prolonged follow-up, patients with PMLBCL presenting at diagnosis with high-risk features or PR response to induction therapy have an encouraging survival with frontline auto-SCT. However, patients who received the transplant after failing the induction regimen have a very poor prognosis and should be tested with other innovative approaches [45]. Dose-dense chemotherapy regimens show excellent results without transplant and the incorporation of rituximab in the treatment have further improved outcomes. Given these excellent results, auto-SCT should not currently be used in patients in first $C R$, but it remains the treatment of choice in relapsed and refractory PMLBCL [46].

\section{Relapsed/refractory primary mediastinal large B-cell lymphoma}

Relapsed or primary refractory PMLBCL is the most challenging situation for clinicians to manage. At progression of the disease dissemination to distant extranodal sites including kidney, adrenal, liver, or central nervous system (CNS) is relatively common but bone marrow involvement is usually absent [6]. Generally, patients with PMLBCL who obtain a CR lasting longer than 24 months are likely to be cured. Studies have shown a plateau at the survival curve beyond 24 months [14].

If early relapse occurs it is a very ominous situation, the same as in primary resistant disease, since that there is no effective option for managing such situations. The outcome is usually disastrous. Central nervous system 
relapse is a rather infrequent complication of $P M L B C L$ relapse, consisting of a difficult therapeutic challenge [47]. It is fatal in most cases and tends to occur 5-12 months after the initial diagnosis [48]. Risk factors for CNS involvement include advanced stage, increased IPI, raised LDH, young age, > 1 extranodal site, B symptoms, low albumin, and retroperitoneal disease [49]. Involvement of specific sites such as the testis, breast, nasal/paranasal sinuses, and bone marrow is associated with a high rate of CNS relapse, which may require CNS prophylaxis. Furthermore, CNS recurrence has been reported in $2-11 \%$ of PMLBL patients, and measures for the CNS prophylaxis have not been discussed for this new disease entity. Central nervous system recurrence has been reported in $2-11 \%$ of PMLBL patients, and measures for CNS prophylaxis have not been discussed for this new disease entity [50]. Managing such relapses could include high-dose methotrexate with highdose cytarabine followed by endocranial RT; however, the outcome is poor.

The outcome of salvage chemotherapy and auto-SCT after the disease returns is generally very poor. There are no defined options regarding how to manage PMLBCL relapse. Patients obtaining PR after front-line therapy could consider the most favourable situation since the remission can be achieved by introduction of RT or, if appropriate, by the addition of high-dose chemotherapy followed by stem cell support. Based on clinical experience, but with no data supported by evidence based medicine, treatment choice in relapse or refractory disease could be any of the salvage regimens defined for DLBCL (ESHAP, DHAP, ICE) followed by high-dose therapy with auto-SCT support. In the retrospective observation by Kuruvilla et al., it was found that refractory/relapsed PMLBCL patients had inferior ORR and survival compared to the same situation DLBCL patients. However, chemo-sensitive relapses of PMLBCL had similar outcome post auto-SCT as DLBCL [51]. Primary refractory disease might be treated with procedures using high-dose chemotherapy followed by auto-SCT as debulking procedures and then by the addition of allo-SCT. However, procedure-related mortality rates and the lack of evidence of procedure efficacy are major limitations. In the era of new molecular insights of the PMLBCL pathogenesis novel agents are warranted (PD-L1, PD-L2, JAK2/STAT molecular pathway inhibitors).

In conclusion, given the new advances in molecular biology of PMLBCL it is becoming increasingly clear that due to its specificity treatment should be more adjusted to its distinct biology profile. Most of the obtained results are based on retrospective studies. The number of prospective studies is increasing, but most of them are with small numbers of patients. Nevertheless, the crucial assumption that the choice of front-line treatment seems to be the critical decision for the management of PMLBCL remains. It is not yet clear which induction approach has the highest benefit for OS, so the guide for the selection of treatment remains an individual centre decision. The third-generation protocols are associated with high toxicity rates and have only a trend to superior outcome, but OS is almost the same as with R-CHOP. As already commented in the text, it can be supposed that DA-EPOCH-R treatment has the highest ranking trend to become a treatment of choice, since it has a confirmation in prospective observation. Nevertheless, results have emerged from the phase II trial and they need to be clarified in larger multicentre prospective randomised phase III trials.

Radiotherapy consolidation remains the treatment modality with the highest obtained controversies throughout the studies. The question of the predictive value of early post-treatment PET/CT remains open because it seems to have low significance (PPV). Dunleavy et al. oppose the routine use of RT when DA-EPOCH-R is to be used. The latest prospective trial of Martelli et al. gives the opportunity of PET/CT scan tailored use of RT, which seems to be the most acceptable treatment modality.

Relapse/refractory disease has poor outcome regardless of the applied treatment modality. Anecdotal sensitive relapses might benefit from the intensified treatments with auto-SCT. Primary refractory disease might undergo allo-SCT after debulking procedure with intensified regimens with auto-SCT, but with limited evidence-based data. New approaches or agencies are warranted, especially in the era of novel molecular insights in the pathogenesis of PMLBCL.

\section{The author declares no conflict of interest.}

\section{References}

1. Miller JB, Variakojis D, Bitran JD, Sweet DL, Kinzie JJ, Golomb HM, Ultmann JE. Diffuse histiocytic lymphoma with sclerosis: a clinicopathological entity frequently causing superior vena caval obstruction. Cancer 1982; 47: 748-56.

2. Trump DL, Mann RB. Diffuse large cell and undifferentiated lymphomas with prominent mediastinal involvement: a poor prognostic subset of patients with non-Hodgkin's lymphoma. Cancer 1982; 50: 277-82

3. Levitt LJ, Aisenberg AC, Harris NL, Linggood RM, Poppema S. Primary non-Hodgkin's lymphoma of the mediastinum. Cancer 1982; 50: 2486-92.

4. Harris NL, Jaffe ES, Stein $H$, et al. A revised European-American classification of lymphoid neoplasms: a proposal from the International Lymphoma Study Group. Blood 1994; 84: 1361-92.

5. Banks PM, Warnke RA. Mediastinal (thymic) large B-cell lymphoma. In: Jaffe ES, Harris NL, Stein H, et al. (eds.). World Health Organization Classification of Tumours: Pathology and Genetics of Tumors of Hematopoietic and Lymphoid Tissues. IARC Press, Lyon 2001; 175-8.

6. Gaulard P, Harris NL, Pileri SA, et al. Primary mediastinal (thymic) large B-cell lymphoma. In: Swerdlow SH, Campo E, Harris NL, et al. (eds.). World Health Organization Classification of Tumors of haematopietic and lymphoid tissues. IARC Press; Lyon 2008; 250-1.

7. Savage KJ, Monti S, Kutok JL, et al. The molecular signature of mediastinal large B-cell lymphoma differs from that of other diffuse large B-cell lymphomas and shares features with classical Hodgkin lymphoma. Blood 2003; 102: 3871-9.

8. Rosenwald A, Wright G, Leroy K, et al. Molecular diagnosis of primary mediastinal B cell lymphoma identifies a clinically favorable subgroup of diffuse large $B$ cell lymphoma related to Hodgkin lymphoma. J Exp Med 2003; 198: 851-62.

9. Green MR, Monti S, Rodig SJ, et al. Integrative analysis reveals selective 9p24.1 amplification, increased PD-1 ligand expression, and further induction via JAK2 in nodular sclerosing Hodgkin lymphoma and primary mediastinal large B-cell lymphoma. Blood 2010; 116: 3268-77. 
10. Steidl C, Gascoyne RD. The molecular pathogenesis of primary mediastinal large B-cell lymphoma. Blood 2011; 118: 2659-69.

11. Twa DD, Chan FC, Ben-Neriah S, et al. Genomic rearrangements involving programmed death ligands are recurrent in primary mediastinal large B-cell lymphoma. Blood 2014; 123: 2062-5.

12. Chan WC, Armitage JO, Gascoyne R, et al. A clinical evaluation of the International Lymphoma Study Group classification of non-Hodgkin's lymphoma. The Non-Hodgkin's Lymphoma Classification Project. Blood 1997; 89: 3909-18.

13. Boleti E, Johnson PW. Primary mediastinal B-cell lymphoma. He matol Oncol 2007; 25: 157-63.

14. Savage KJ, Al-Rajhi N, Voss N, Paltiel C, Klasa R, Gascoyne RD, Connors JM. Favourable outcome of primary mediastinal large B-cell lymphoma in a single institution: the British Columbia experience. Ann Oncol 2006; 17: 123-30.

15. Todeschini G, Ambrosetti A, Meneghini V, et al. Mediastinal large B-cell lymphoma with sclerosis: a clinical study with of 21 patients. J Clin Oncol 1990; 8: 804-8.

16. Hamlin PA, Portlock CS, Straus DJ, et al. Primary mediastinal B-cell lymphoma: optimal therapy and prognostic factor analysis in 141 consecutive patients treated in Memoria Sloan Kettering from 1980 to 1999. Br J Haematol 2005; 130: 691-99.

17. Haioun C, Gaulard P, Roudot-Thoraval F, et al. Mediastinal diffuse large B-cell lymphoma with sclerosis: a condition with a poor prognosis. Am J Clin Oncol 1989; 12: 425-9.

18. Todeschini G, Secchi S, Morra E, et al. Primary mediastinal large B-cell lymphoma (PMLBCL): long-term results from a retrospective multicentre Italian experience in 138 patients treated with $\mathrm{CHOP}$ or MACOP-B/VACOP-B. Br J Cancer 2004; 90: 372-6.

19. Bertini M, Orsucci L, Vitolo U, et al. Stage II large B-cell lymphoma with sclerosis treated with MACOP-B. Ann Oncol 1991; 2: 733-7.

20. Lazzarino M, Orlandi E, Paulli M, et al. Primary mediastinal B-cell lymphoma with sclerosis. An aggressive tumor with distinctive clinical and pathologic features. J Clin Oncol 1993; 11: 2306-13.

21. Falini B, Venturi S, Martélli M, et al. Mediastinal large B-cell lymphoma. Clinical and immunohistological findings in 18 patients treated with different third-generation regimens. Br J Haematol 1995; 89: 780-9.

22. Cazals-Hatem D, Lepage E, Brice P, et al. A clinicopathologic study of 141 cases compared with 916 non-mediastinal large B-cell lymphomas, a GELA ("Groupe d'Etude des Lymphomes de l'Adulte") study. Am J Surg Pathol 1996; 20: 877-88.

23. Lazzarino M, Orlandi E, Paulli M, et al. Treatment outcome and prognostic factors for primary mediastinal (Thymic) B-cell lymphoma: a multicenter study of 106 patients. J Clin Oncol 1997; 15 1646-53.

24. Zinzani PL, Martelli M, Bertini M, et al.; International Extranodal Lymphoma Study Group (IELSG). Induction chemotherapy strate gies for primary mediastinal large B-cell lymphoma with sclerosis: a retrospective multinational study on 426 previously untreated patients. Haematologica 2002; 87: 1258-64.

25. Zinzani PL, Stefoni V, Finolezzi E, et al. Rituximab combined with MACOP-B or VACOP-B and radiation therapy in primary mediastinal large B-cell lymphoma: a retrospective study. Clin Lymphoma Myeloma 2009; 9: 381-5.

26. Gutierrez M, Chabner BA, Pearson D, Steinberg SM, Jaffe ES, Cheson BD, Fojo A, Wilson WH. Role of a doxorubicin-containing regimen in relapsed and resistant lymphomas: an 8-year follow-up study of EPOCH. J Clin Oncol 2000; 18: 3633-42.

27. Legha SS, Benjamin RS, Mackay B, et al. Reduction of doxorubicin cardiotoxicity by prolonged continuous intravenous infusion. Ann Intern Med 1982; 96: 133-9.

28. Wilson WH, Grossbard ML, Pittaluga S, et al. Dose-adjusted EPOCH chemotherapy for untreated large B-cell lymphomas: a pharmacodynamic approach with high efficacy. Blood 2002; 99: 2685-93.

29. Wilson WH, Bryant G, Bates S, et al. EPOCH chemotherapy: toxicity and efficacy in relapsed and refractory non-Hodgkin's lymphoma. J Clin Oncol 1993; 11: 1573-82.

30. Pfreundschuh M, Trümper L, Osterborg A, et al.; MabThera International Trial Group. CHOP-like chemotherapy plus rituximab versus CHOP-like chemotherapy alone in young patients with good-prognosis diffuse large-B-cell lymphoma: A randomised controlled trial by the MabThera International Trial (MInT) Group. Lancet Oncol 2006; 7: 379-91

31. Rieger M, Osterborg A, Pettengell R, et al. MabThera Internationa Trial (MInT) Group. Primary mediastinal B-cell lymphoma treated with CHOP-like chemotherapy with or without rituximab: Results of the MabThera International Trial Group study. Ann Oncol 2011; 22: 664-70.

32. Vassilakopoulos TP, Pangalis GA, Katsigiannis A, et al. Rituximab, cyclophosphamide, doxorubicin, vincristine, and prednisone with or without radiotherapy in primarymediastinal large B-cell lymphoma: the emerging standard of care. Oncologist 2012; 17: 239 49

33. Ahn HK, Kim SJ, Yun J, et al. Improved treatment outcome of primary mediastinal large B-cell lymphoma after introduction of rituximab in Korean patients. Int J Hematol 2010; 91: 456-63.

34. Schneider T, Tóth E, Lovey J, et al. Standard CHOP immune-chemotherapy for primary mediastinal lymphomas. Orv Hetil 2011; 152 735-42.

35. Xu LM, Fang $H$, Wang WH, et al. Prognostic significance of rituximab and radiotherapy for patients with primary mediastinal large B-cell lymphoma receiving doxorubicin-containing chemotherapy. Leuk Lymphoma 2013; 54: 1684-90.

36. Wang BJ, Cen XN, Qiu ZX, et al. Clinical characteristics and longterm follow-up of 29 patients with primary mediastinal large B cell lymphoma. Zhongguo Shi Yan Xue Ye Xue Za Zhi 2014; 22: 1596-602.

37. Dunleavy K, Pittaluga S, Janik J, et al. The addition of rituximab to dose adjusted (DA)-EPOCH obviates the need for radiation in the treatment of primary mediastinal large B-cell lymphoma ( $\mathrm{PMBL}$ ): A prospective study of 58 patients [Abstract]. Ann Oncol 2008; 19 suppl 4: iv96.

38. Dunleavy K, Pittaluga S, Maeda LS, et al. Dose-adjusted EPOCH-rituximab therapy in primary mediastinal B-cell lymphoma. N Engl J Med 2013; 368: 1408-16.

39. Purroy N, Bergua J, Gallur L, et al. Long-term follow-up of dose-adjusted EPOCH plus rituximab (DA-EPOCH-R) in untreated patients with poorprognosis large B-cell lymphoma. A phase II study conducted by the Spanish PETHEMA Group. Br J Haematol 2015; 169: 188-98.

40. Avigdor A, Sirotkin T, Kedmi M, et al. The impact of R-VACOP-B and interim FDG-PET/CT on outcome in primary mediastinal large B cell lymphoma. Ann Hematol 2014; 93: 1297-304.

41. Zinzani PL, Broccoli A, Casadei B, et al. The role of rituximab and positron emission tomography in the treatment of primary mediastinal large B-cell lymphoma: experience on 74 patients. Hematol Oncol 2014 Sep 25; doi: 10.1002/hon.2172.

42. Kirk R. Hematological cancer: new standard of care? Nat Rev Clin Oncol 2013; 10: 305

43. Filippi AR, Piva C, Giunta F, et al. Radiation therapy in primary mediastinal B-cell lymphoma with positron emission tomography positivity after rituximab chemotherapy. Int J Radiat Oncol Biol Phys 2013; 87: 311-6.

44. Martelli M, Ceriani L, Zucca E, et al. [18F]fluorodeoxyglucose pos itron emission tomography predicts survival after chemoimmunotherapy for primary mediastinal large B-cell lymphoma: results of the International Extranodal Lymphoma Study Group IELSG-26 Study. J Clin Oncol 2014; 32: 1769-75.

45. Rodríguez J, Conde E, Gutiérrez A, et al. Primary mediastinal large cell lymphoma (PMBL): frontline treatment with autologous stem cell transplantation (ASCT). The GEL-TAMO experience. Hematol Oncol 2008; 26: 171-8.

46. Poiré X, van Besien K. Autologous transplant for primary mediastinal B-cell lymphoma. Expert Rev Hematol 2009; 2: 31-6.

47. Stefoni V, Broccoli A, Pellegrini C, Derenzini E, Fina M, Zinzani PL. CNS recurrence of primary mediastinal large B-cell after complete remission. J Neurooncol 2009; 95: 135-9.

48. Hill QA, Owen RG. CNS prophylaxis in lymphoma: who to target and what therapy to use. Blood Rev 2006; 20: 319-32.

49. Hollender A, Kvaloy S, Nome O, Skovlund E, Lote K, Holte H. Central nervous system involvement following diagnosis of non-Hodgkin's lymphoma: a risk model. Ann Oncol 2002; 13: 1099-107. 
50. Sasaki M, Sugimoto K. Central nervous system recurrence in the primary mediastinal large B-cell lymphoma: treatment. In: Tumors of the central nervous system. Volume 9. Hayat MA (ed.). Springer; 2012; 21-7.

51. Kuruvilla J, Pintilie M, Tsang R, Nagy T, Keating A, Crump M. Salvage chemotherapy and autologous stem cell transplantation are inferior for relapsed or refractory primary mediastinal large B-cell lymphoma compared with diffuse large B-cell lymphoma. Leuk Lymphoma 2008; 49: 1329-36.

\section{Address for correspondence}

Ivan Petković

Oncology Clinic

Bulevar Dr Zorana Đinđića 48

18000 Niš, Serbia

e-mail: ivan76.unsu@yahoo.com

Submitted: 9.09 .2014

Accepted: 23.01.2015 